\title{
Effect of Hydrogen Bonding on the Deformation Frequencies of the Hydroxyl Group in Alcohols*
}

\author{
A. V. Stuart $\dagger$ and G. B. B. M. Sutherland \\ Department of Physics, University of Michigan, Ann Arbor, Michigan
}

(Received June 13, 1955)

\begin{abstract}
The effects of hydrogen bonding on the infrared spectra of aliphatic alcohols have been studied in the range 4000 to $350 \mathrm{~cm}^{-1}$. Twenty-six alcohols were investigated of which 10 were primary, 7 were branched primary, 6 were secondary, and 3 were tertiary. Attention was concentrated on the region of the spectrum between $1500 \mathrm{~cm}^{-1}$ and $350 \mathrm{~cm}^{-1}$ where the deformation vibrations of the $\mathrm{OH}$ group occur. Spectra were obtained of the alcohols (a) in dilute solution in nonpolar solvents, (b) in the liquid state, and (c) in the vapor state, the degree of association being followed by observation of the well-known effect of hydrogen bonding in the $\mathrm{OH}$ stretching vibration near 3.0 $\mathrm{\mu}$. Methanol, ethanol, and hexanol-1 were also investigated after deuteration of the OII group. All the alcohols exhibited a broad diffuse association band with a maximum near $650 \mathrm{~cm}^{-1}$. This has been assigned to the out-of-plane deformation vibration of the $\mathrm{H}$ atom in the $\mathrm{COH}$ group. All the alcohols also exhibited a broad association band which usually had 2 maxima near $1410 \mathrm{~cm}^{-1}$ and $1330 \mathrm{~cm}^{-1}$. This band (which appears to have been missed by previous workers) is assigned to the in-plane deformation vibration of the $\mathrm{H}$ atom in the $\mathrm{COH}$ group. The corresponding monomeric band varies between $1200 \mathrm{~cm}^{-1}$ and $1330 \mathrm{~cm}^{-1}$ in undeuterated alcohols and between $870 \mathrm{~cm}^{-1}$ and $930 \mathrm{~cm}^{-1}$ in deuterated alcohols. There is in addition a very narrow association band which lies near $1100 \mathrm{~cm}^{-1}$ in primary and secondary alcohols and near $1165 \mathrm{~cm}^{-1}$ in tertiary alcohols. This is assigned to the effect of hydrogen bonding on the skeletal vibrations, which involve stretching of the $\mathrm{CO}$ bond. It appears that the effects of hydrogen bonding on the deformation motions of the $\mathrm{OH}$ group are quite complex and that steric effects, rotational isomerism, and interaction with $\mathrm{C}-\mathrm{H}$ deformation frequencies may all be involved to some extent.
\end{abstract}

A LTHOUGH extensive studies have been made of the effects of hydrogen bonding on vibrational spectra, the majority of the work has been concentrated on a very limited region of the infrared spectrum between 1 and $4 \mu$, which includes only the stretching (valency) vibrations of the bonded hydrogen. ${ }^{1}$ The deformation vibrations have received very scant attention and indeed even the assignment of the deformation frequencies of the $\mathrm{OH}$ and $\mathrm{NH}$ groups is uncertain in many molecules. ${ }^{2}$ Since even weak intermolecular bonding may be expected to have quite marked effects on deformation frequencies, the changes in the deformation frequencies of $\mathrm{H}$ atoms involved in hydrogen bonding should be easily recognized and provide a means of identifying these particular modes of vibration in the spectrum of a large molecule containing an $\mathrm{OH}$ or $\mathrm{NH}$ group. An alternative way to identify hydrogenic frequencies is to investigate the effects of deuteration on the spectrum. Neither method is satisfactory by itself, but a combination of the two allows crosschecks to be made. With this in mind a systematic study has been made of the effects of hydrogen bonding on the infrared spectra of a series of undeuterated and deuterated alcohols between $6 \mu$ and $25 \mu$, i.e. the region in which hydrogenic deformation vibrations are always found.

* Presented in part before the "Réunion internationale de spectroscopie moléculaire," Paris, 1953, and briefly reported J. phys. radium 15, 321 (1954).

† Now with Koninklijke/Shell Laboratorium, Amsterdam, Netherlands.

${ }^{1}$ Reviews of this work have been given by: M. M. Davies, Ann. Repts. Progr. Chem. (Chem. Soc. London) 43, 3 (1946); L. Kellner, Repts. Progr. Phys. XV, 1 (1952).

${ }^{2}$ G. B. B. M. Sutherland, Discussions Faraday Soc. 9, 261 (1950).

\section{EXPERIMENTAL}

The alcohols were investigated in solution in $\mathrm{CCl}_{4}$ or $\mathrm{CS}_{2}$ at various concentrations. The degree of hydrogen bonding was determined from the relative intensities of the well-known $\mathrm{OH}$ stretching vibrations at $3640 \mathrm{~cm}^{-1}$ (free $\mathrm{OH}$ ) and $3300 \mathrm{~cm}^{-1}$ (bonded $\mathrm{OH}$ ). Many of the alcohols were also studied in the vapor state, and the spectra of a few were obtained after deuteration of the $\mathrm{OH}$ group to an OD group. The specific influence of the solvent was checked in a study of 3 nonpolar and 3 polar solvents; the results of this work have been published elsewhere. ${ }^{3}$

The principal spectrometer used was a Perkin-Elmer Model 21 double-beam instrument, equipped with a rocksalt prism, thus allowing spectra to be obtained out to $650 \mathrm{~cm}^{-1}(15.4 \mu)$. Frequencies down to $350 \mathrm{~cm}^{-1}$ $(28.6 \mu)$ were studied using a Perkin Elmer Model $12 \mathrm{C}$ single-beam spectrometer, equipped with a KRS-5 prism and a $\mathrm{LiF}$ reststrahlen plate. The double-beam arrangement allowed compensation of the absorption due to the solvent. This was achieved by placing in the reference beam a cell containing an amount of pure solvent exactly equal to the amount of solvent in the sample solution. A cell of variable thickness ${ }^{4}$ was used for this purpose.

In some cases it was of interest to determine relatively small spectral changes, due to a change in concentration, in regions where other bands of medium but constant intensity also occurred. To disentangle the various bands a double compensation method was successfully applied. In this method one places in the first beam a

${ }^{3}$ A. V. Stuart, J. Chem. Phys. 21, 1115 (1953).

${ }^{4}$ A. V. Stuart, J. Opt. Soc. Am. 43, 212 (1953). 


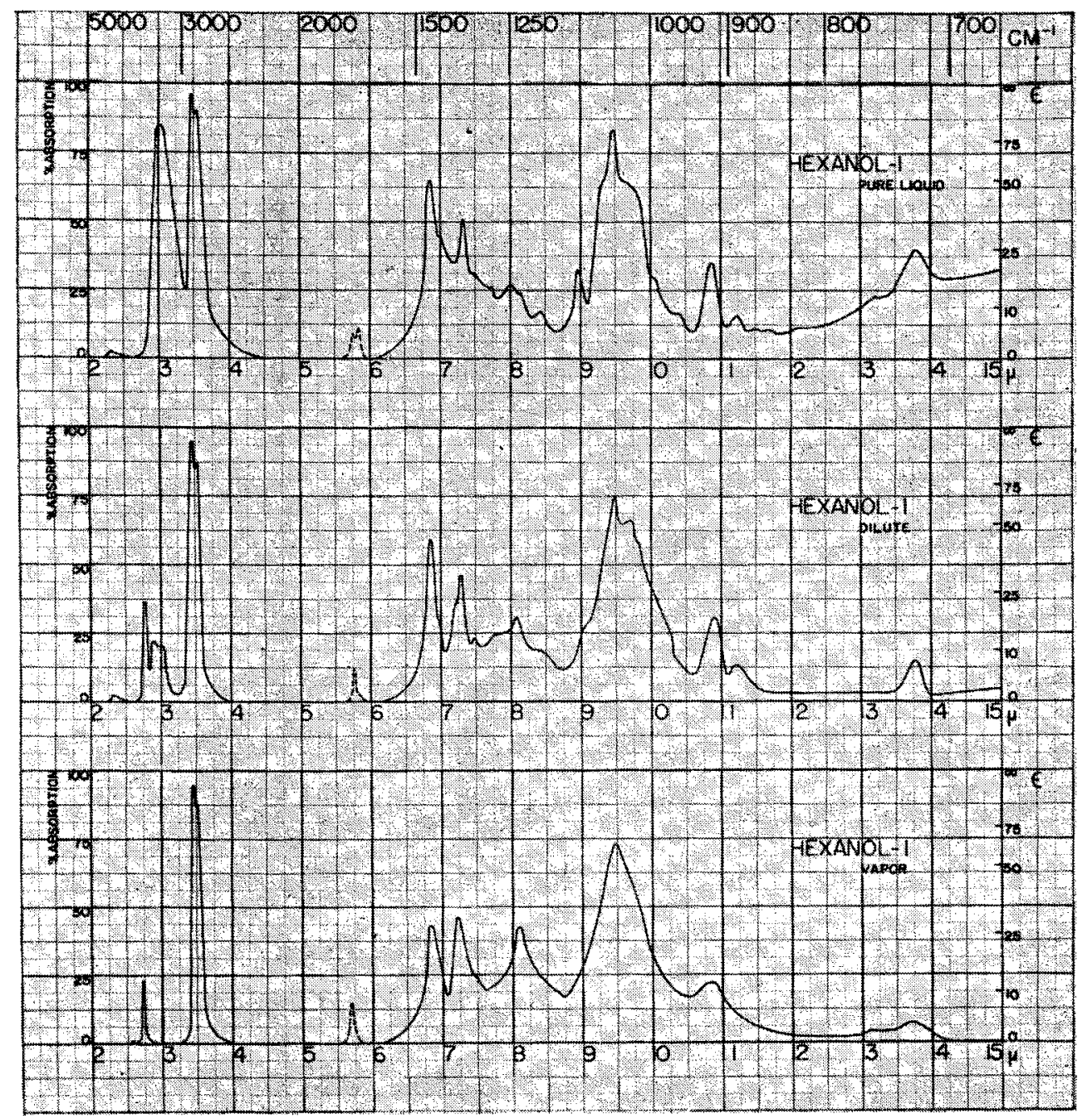

Frg. 1. Hexanol-1 as pure liquid, 0.1 molar solution in nonpolar solvent, and as vapor. thin cell containing a solution of high concentration and in the other beam a thick cell with a solution of low concentration, such that the amounts of solute are exactly the same in both cells. The thinner cell is then combined with a variable thickness cell containing the pure solvent in order to compensate for the difference in solvent in the fixed cells. The double-beam spectrometer thus records only the difference in absorption of the solute molecules due to the change in concentration. This method was especially valuable in the region between 1500 and $1250 \mathrm{~cm}^{-1}$ where the $\mathrm{CH}$ deformation frequencies occur.

Although the main portion of the work was concerned with the dependence on concentration of the spectra of alcohols in solution, the effect of vaporization has also been studied. For the lower alcohols a conventional gas cell could be used, but Butanol-1 and higher alcohols had to be heated in order to give sufficiently high vapor pressures. A brass tube, $3 \frac{1}{2}$ in. long, enclosed in a furnace, served in these cases. The temperature never had to be raised very high. For instance, the spectrum of Decanol-1, which has a boiling point of $231^{\circ} \mathrm{C}$, was obtained at $140^{\circ} \mathrm{C}$. At this temperature the vapor pressure is about $2 \mathrm{~cm} \mathrm{Hg}$, the equivalent of the concentration which was found to give the best results for the solution spectra.

Twenty-six alcohols were studied, of which 10 were n-primary, 7 were branched primary, 6 were secondary, and 3 were tertiary. Three of the $n$-primary alcohols, viz., methanol, ethanol, and hexanol, were also studied in the deuterated form. The alcohols used were mostly obtained from commercial suppliers of pure chemicals and were purified by further distillation in a column of 21 theoretical plates. The principal impurity was water, which could be detected by means of the well-known band at $6.1 \mu$. In some cases, small amounts of ketone or aldehyde impurity also had to be removed. These were detected through the $\mathrm{CO}$ band near $5.7 \mu$. Only in the case of $n$-hexanol was it impossible to reduce the impurity to a negligible amount. The impurity bands have been ignored in all of the spectral curves reproduced here except that of $n$-hexanol (Fig. 1), and even there it will be seen that the amount of residual impurity is very small. The sample of 2-methyl-pentanol-1 seemed to 
Fig, 2. Methanol as pure liquid, 0.1 molar solution in nonpolar solvent, and as vapor.

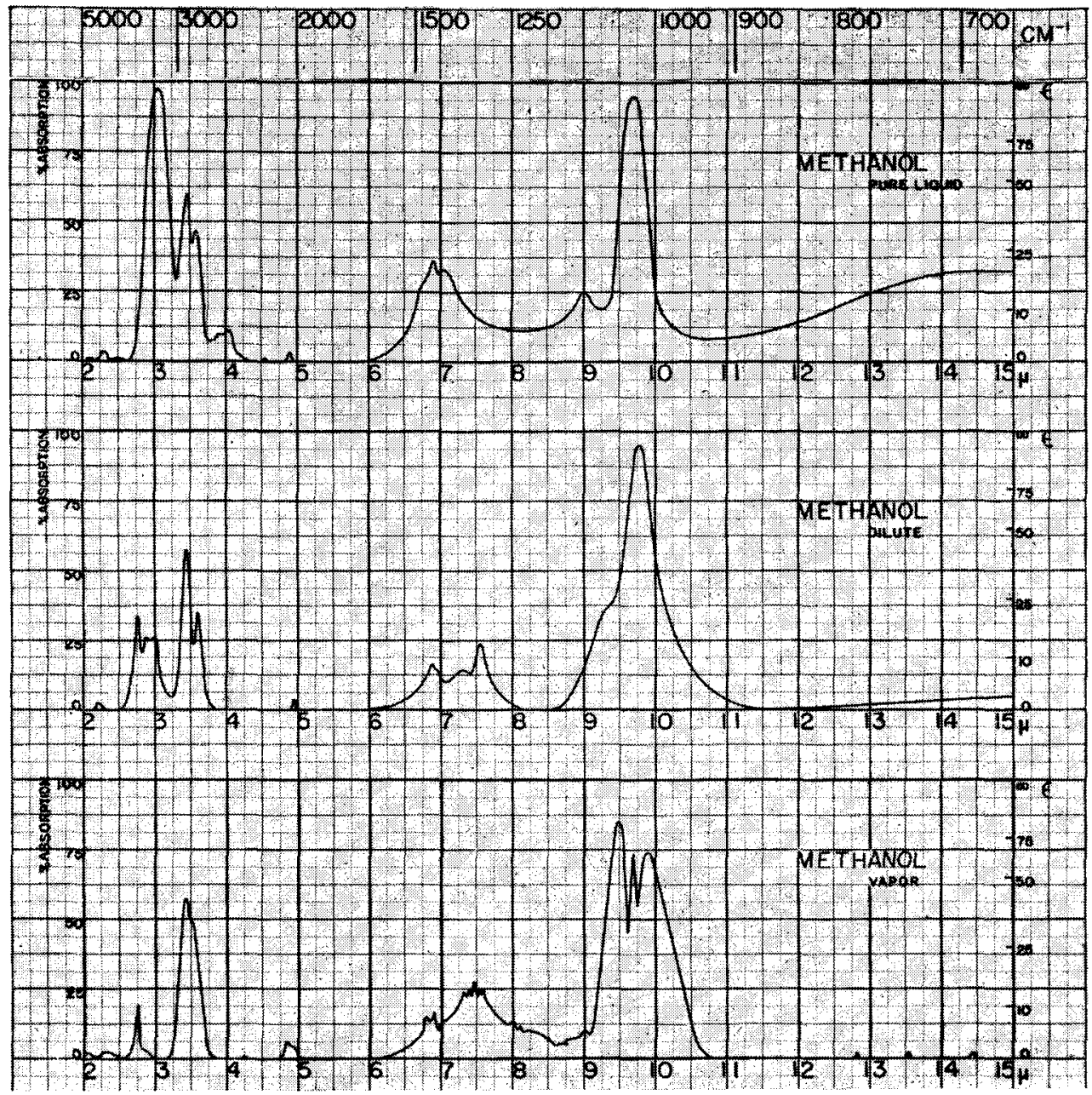

spectrum extending from 2 to $15 \mu$. This is the scale used in Figs. 1 to 5 and in Fig. 7, although for convenience a nonlinear wave number scale is also added at the top of each diagram. In discussing the results, we use a linear wave number scale, and this scale is also used over the limited regions of the spectrum shown in Figs. 8 and 9. The vertical scale is linear in percent absorption, but in Figs. 1 to 6 and 8 and in Fig. 7, the observations have all been reduced (assuming Beer's law) to a common base in which the number of absorbing molecules in the path is the same, i.e. the product of molar concentration $(c)$ and cell length $(l)$ is very close to $9 \times 10^{-6} \mathrm{~mole} / \mathrm{cm}^{2}$. On the right-hand side of these 8 figures will be found an approximate (nonlinear) scale of extinction coefficients in units of $10^{3}$ mole ${ }^{-1} \mathrm{~cm}^{2}$ where the extinction coefficient $\epsilon$ is defined by the equation

$$
\epsilon=\frac{1}{c l} \log _{10} \frac{I_{0}}{I}
$$

In presenting the observations in graphical form it is convenient to use a linear wavelength scale, since this is the scale on our Perkin-Elmer Model 21 spectrometer, and it is the most practical scale on which to show a
$I_{0}$ and $I$ being respectively the incident and transmitted intensity. 


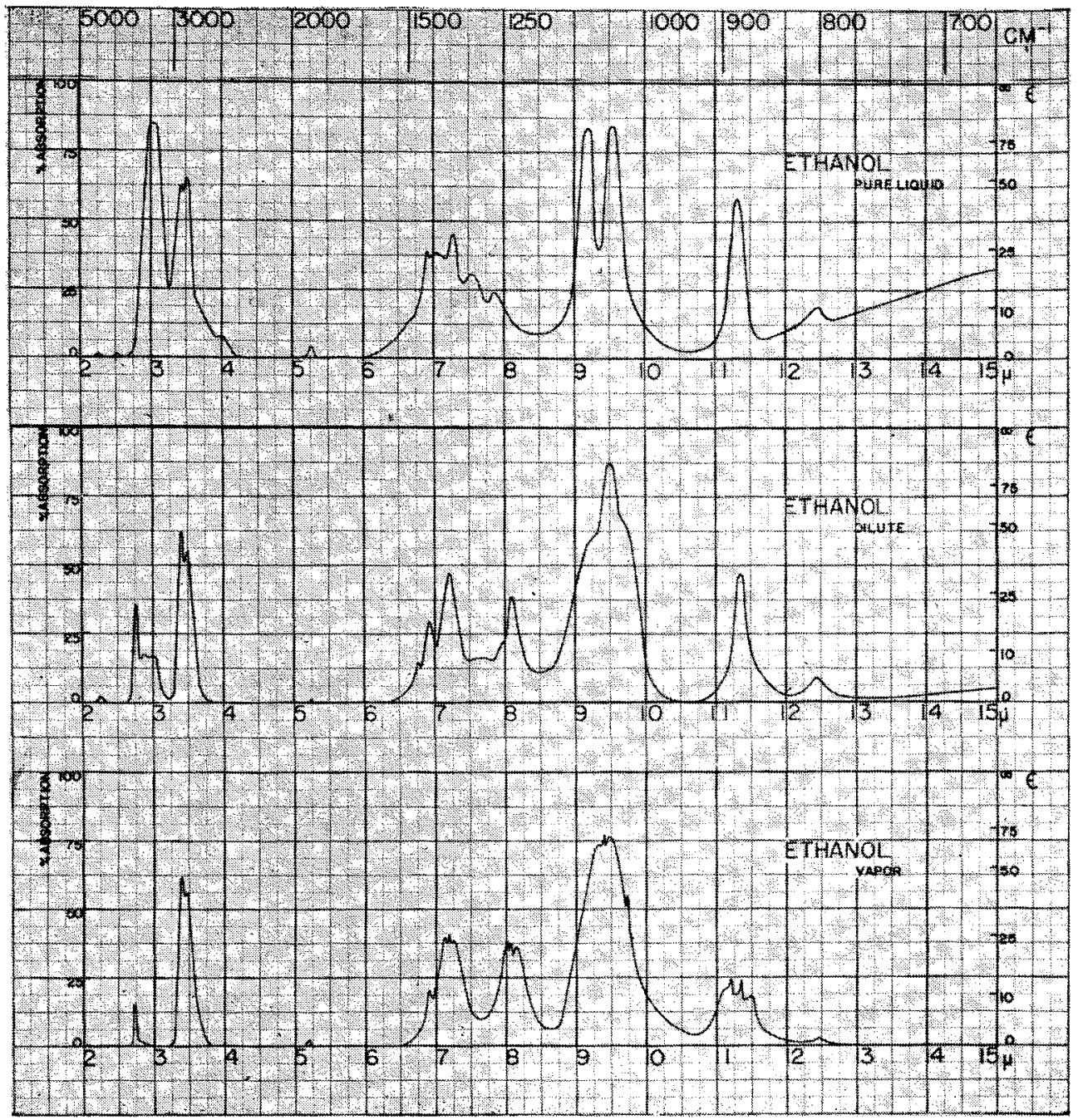

FIG, 3. Ethanol as pure liquid, 0.1 molar solution in nonpolar solvent, and as vapor.

\section{Normal Primary Alcohols}

The spectra of a series of normal primary alcohols from methanol to decanol-1 were obtained (a) as pure liquids, (b) in solution sufficiently dilute to break up most of the hydrogen bonding, and (c) in the vapor state. The first 6 of these are illustrated in Figs. 1 to 6. The higher alcohols show spectra very similar to those of hexanol-1. In comparing the changes which occur for each alcohol as one proceeds from the liquid state, through dilute solution, to the vapor, certain features are found to be common to all of them. Thus, associated with the disappearance of the well-known bonded $\mathrm{OH}$ stretching vibration near $3 \mu\left(3300 \mathrm{~cm}^{-1}\right)$ one finds that absorption bands, near $7 \mu\left(1410 \mathrm{~cm}^{-1}\right), 7.5 \mu(1330$ $\left.\mathrm{cm}^{-1}\right), 9.0 \mu\left(1110 \mathrm{~cm}^{-1}\right)$, and $15 \mu\left(670 \mathrm{~cm}^{-1}\right)$ decrease in intensity. Such bands will be called association bands. On the other hand, the appearance of the free $\mathrm{OH}$ band near $2.77 \mu\left(3640 \mathrm{~cm}^{-1}\right) \ddagger$ is accompanied in many cases

$¥$ This band appears at $3644 \mathrm{~cm}^{-1}$ in a dilute $\mathrm{CCl}_{4}$ solution, and at $3627 \mathrm{~cm}^{-1}$ in $\mathrm{CS}_{2}$; an "average" value of $3640 \mathrm{~cm}^{-1}$ is used since only the general effects are under discussion here. by the appearance (or a marked increase in the intensity) of a band in the neighborhood of $8 \mu$ (1250 $\mathrm{cm}^{-1}$ ). It will be observed that this monomer band has an exceptionally high value $\left(1340 \mathrm{~cm}^{-1}\right)$ in methanol, and a rather high and constant value of about 1290 $\mathrm{cm}^{-1}$ in octanol-1, nonanol-1, and decanol-1. For the other $6 n$-primary alcohols, it varies in position between $1200 \mathrm{~cm}^{-1}$ and $1255 \mathrm{~cm}^{-1}$. These changes are indicated diagrammatically in Fig. 10. Such bands will be designated as monomer bands. There is also an indication of a monomer band near $1080 \mathrm{~cm}^{-1}$ in methanol, ethanol, and hexanol-1 and in ethanol and hexanol near $1020 \mathrm{~cm}^{-1}$.

It should be noted that the association bands near $1410 \mathrm{~cm}^{-1}, 1330 \mathrm{~cm}^{-1}$, and $650 \mathrm{~cm}^{-1}$ (especially the last) are rather diffuse, whereas that near $1110 \mathrm{~cm}^{-1}$ is relatively sharp and well defined. It is particularly hard to determine the contours of the bands near $1410 \mathrm{~cm}^{-1}$ and $1330 \mathrm{~cm}^{-1}$ because of the neighboring $\mathrm{CH}_{2}$ and $\mathrm{CH}_{3}$ deformation bands at $1460 \mathrm{~cm}^{-1}$ and $1375 \mathrm{~cm}^{-1}$. Accordingly these were further investigated by the double compensation method described above, and the results 
FIG. 4. Propanol-1 as pure liquid, 0.1 molar solution in nonpolar solvent, and as vapor.

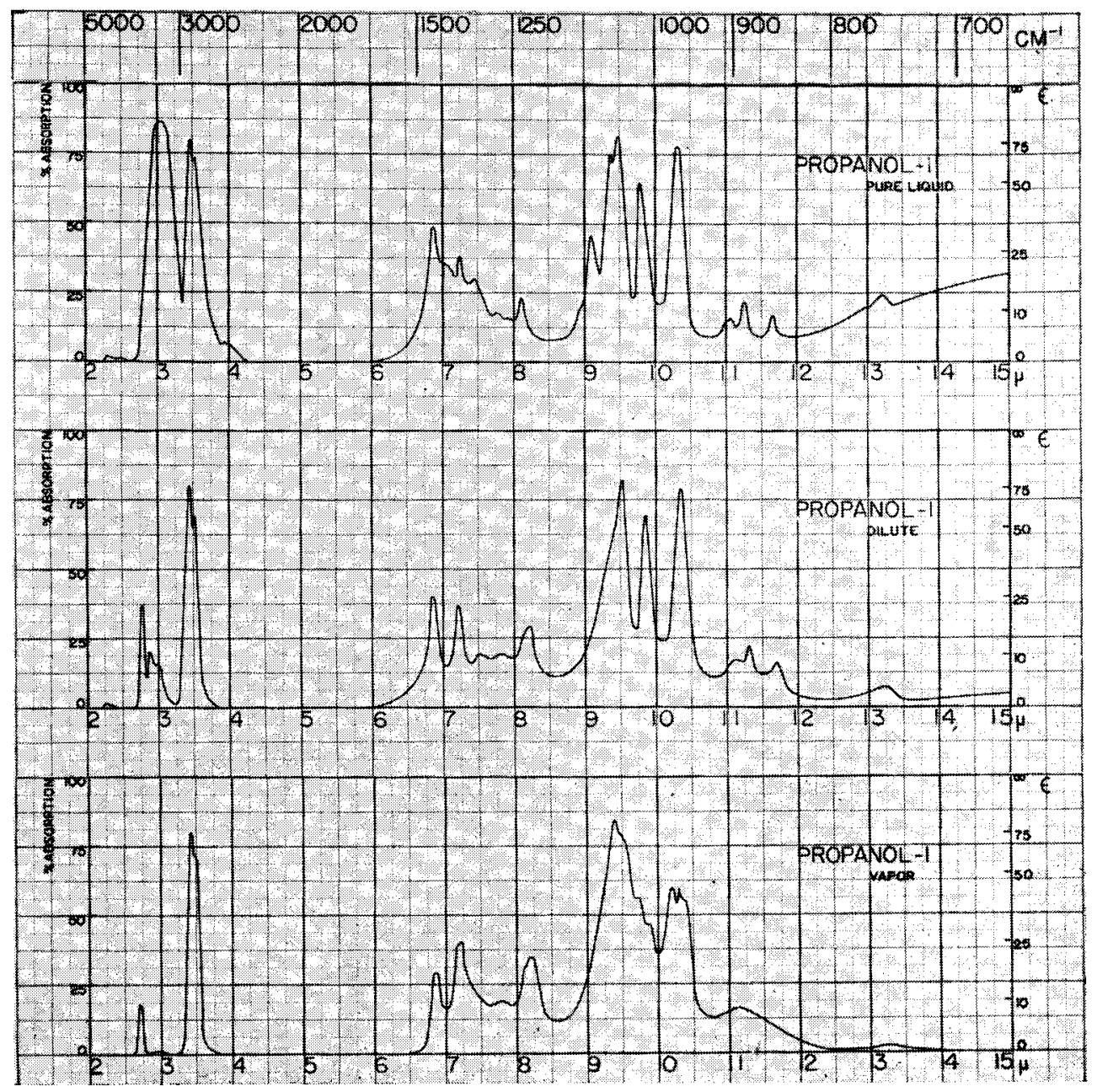

are illustrated in Fig. 8 for the first 4 members of this series of alcohols. The case of methanol is apparently an exception, since the association band near $1330 \mathrm{~cm}^{-1}$ is missing and a very narrow band near $6.9 \mu\left(1445 \mathrm{~cm}^{-1}\right)$ shows the behavior of an association band, a feature which is absent from all the other alcohols.

Two alcohols (propanol-1 and butanol-1) were investigated in solution at different temperatures in the range of concentration used for the room temperature studies. The results are essentially the same as reported above, if we assume that a rise in temperature breaks up association through hydrogen bonds.

\section{Other Primary Alcohols}

Seven other primary alcohols were investigated which had branching in the hydrocarbon chain. The results (which are included diagrammatically in Fig. 10) were on the whole very similar to those obtained for the normal primary alcohols. Thus association bands again appeared at $1410 \mathrm{~cm}^{-1}, 1330 \mathrm{~cm}^{-1}, 1110 \mathrm{~cm}^{-1}$, and 670 $\mathrm{cm}^{-1}$, and as the association was broken up a monomer band appeared in the neighborhood of $1200 \mathrm{~cm}^{-1}$ (except for 5-methyl-hexanol-1 where it was at $\left.1255 \mathrm{~cm}^{-1}\right)$.
However, in these samples another monomer band was very definitely established near $1100 \mathrm{~cm}^{-1}$. It should be added that the $1110 \mathrm{~cm}^{-1}$ and $1100 \mathrm{~cm}^{-1}$ bands were both rather weak. This is illustrated for 2-methylbutanol-1 in Fig. 9.

\section{Secondary Alcohols}

Six secondary alcohols were investigated. The results (also shown diagrammatically in Fig. 10) are very similar to those found for the branched chain primary alcohols, the main difference being that the positions of the bands near $1330 \mathrm{~cm}^{-1}$ and $1230 \mathrm{~cm}^{-1}$ are more variable than in the branched primary alcohols. The bands near $1110 \mathrm{~cm}^{-1}$ and $1070 \mathrm{~cm}^{-1}$, however, are now considerably stronger than in the previous cases. This is illustrated for butanol-2 in Fig. 9.

\section{Tertiary Alcohols}

Three tertiary alcohols were investigated. These show very different characteristics, the only feature in common with the primary and secondary alcohols being the association band at $1410 \mathrm{~cm}^{-1}$. However, there is no association band near $1330 \mathrm{~cm}^{-1}$. Instead one finds a 


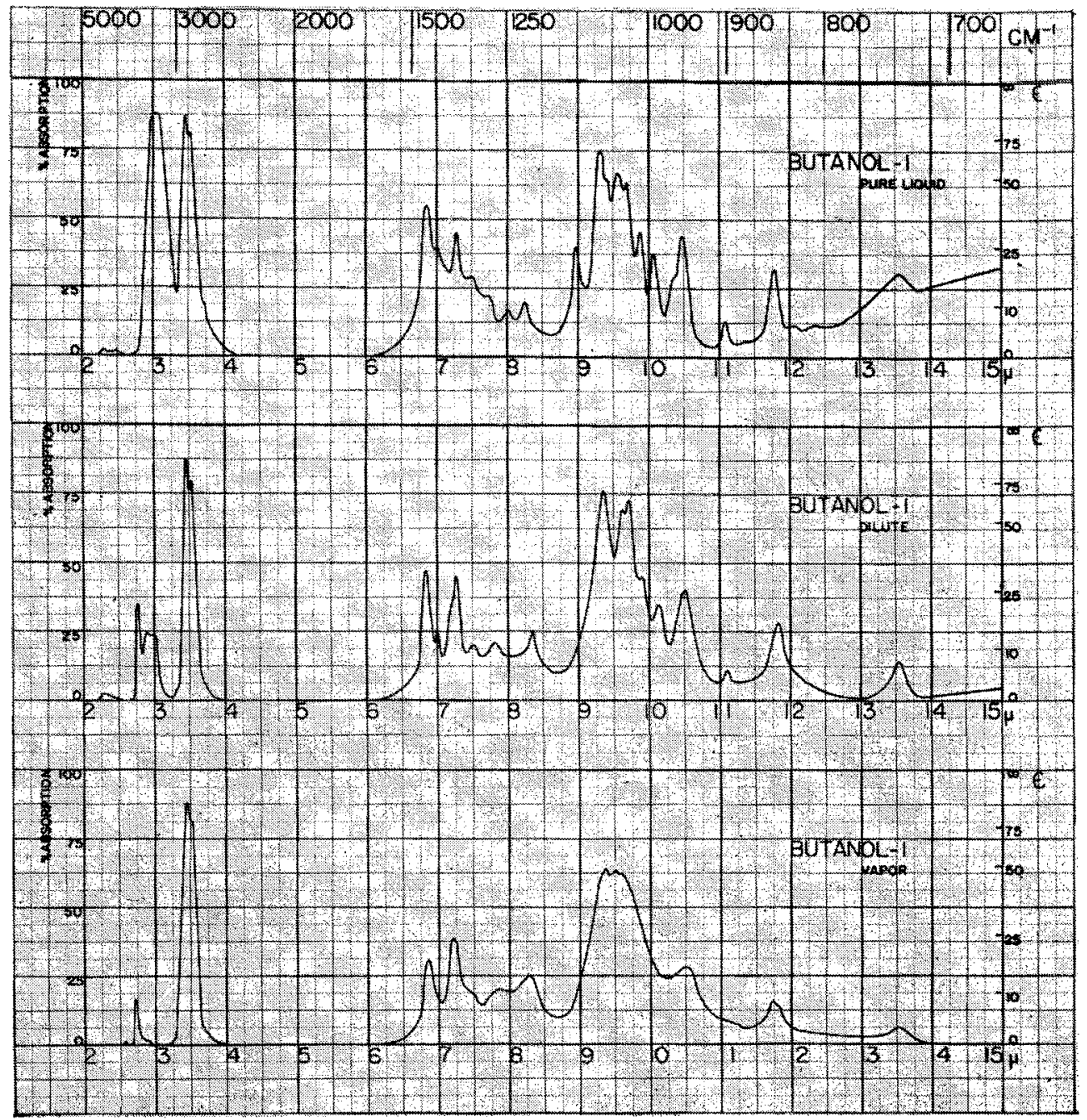

Frg. 5. Butanol-1 as pure liquid, 0.1 molar solution in nonpolar solvent, and as vapor. monomer band near $1315 \mathrm{~cm}^{-1}$. This situation is very similar to the case of methanol mentioned earlier. There is no monomer band between $1200 \mathrm{~cm}^{-1}$ and $1250 \mathrm{~cm}^{-1}$, and the association band found near $1110 \mathrm{~cm}^{-1}$ in other alcohols appears to have moved to around $1160 \mathrm{~cm}^{-1}$ while its companion monomer band has also moved to about $1140 \mathrm{~cm}^{-1}$. Here the $1160 \mathrm{~cm}^{-1}$ and $1140 \mathrm{~cm}^{-1}$ bands are rather strong. As an example of these results the spectrum of 2-methyl-butanol-2 is given in Fig. 9.

\section{Deuterated Alcohols}

The effects of dilution were also studied for methanol, ethanol, and hexanol-1 in which the $\mathrm{OH}$ group had been converted to an OD group. The results for methanol are illustrated in Fig. 7, which includes the corresponding spectra for undeuterated methanol. It will be seen that all of the association and monomer bands found in the undeuterated methanol are absent from the deuterated methanol. The $\mathrm{OH}$ stretching frequencies near $3 \mu$ are replaced by $\mathrm{OD}$ stretching frequencies near $4 \mu$. More specifically, the OH monomer band at $3640 \mathrm{~cm}^{-1}$ be- comes an OD monomer band at $3.75 \mu\left(2670 \mathrm{~cm}^{-1}\right)$. The corresponding association band has a well-marked doublet structure with maxima at $3.9 \mu\left(2565 \mathrm{~cm}^{-1}\right)$ and $4.15 \mu\left(2410 \mathrm{~cm}^{-1}\right)$. These bands were used as a measure of the degree of dissociation in the same way that corresponding bands were used in the undeuterated alcohols. In the region of deformation frequencies, 3 association bands were found, viz. 1225, 930, and 470 $\mathrm{cm}^{-1}$. The latter two bands are wide and diffuse and resemble in this respect the association bands of undeuterated methanol found at 1410, 1330, and 650 $\mathrm{cm}^{-1}$. The association band at $1225 \mathrm{~cm}^{-1}$ is relatively sharp and not so intense. Only one monomer band was found in $D$-methanol. It is quite an intense band at 870 $\mathrm{cm}^{-1}$, i.e. on the long wave edge of the $950 \mathrm{~cm}^{-1}$ association band.

The results for $D$-ethanol and $D$-hexanol-1 were quite similar in many respects and are illustrated diagrammatically in Fig. 11. The association band found near $930 \mathrm{~cm}^{-1}$ in $\mathrm{CH}_{3} \mathrm{OD}$ appears at higher frequencies in $\mathrm{C}_{2} \mathrm{H}_{5} \mathrm{OD}\left(955 \mathrm{~cm}^{-1}\right)$ and $\mathrm{C}_{6} \mathrm{H}_{11} \mathrm{OD}\left(960 \mathrm{~cm}^{-1}\right)$. The 
Fig. 6. Pentanol-1 as pure liquid, 0.1 molar solution in nonpolar solvent, and as vapor.

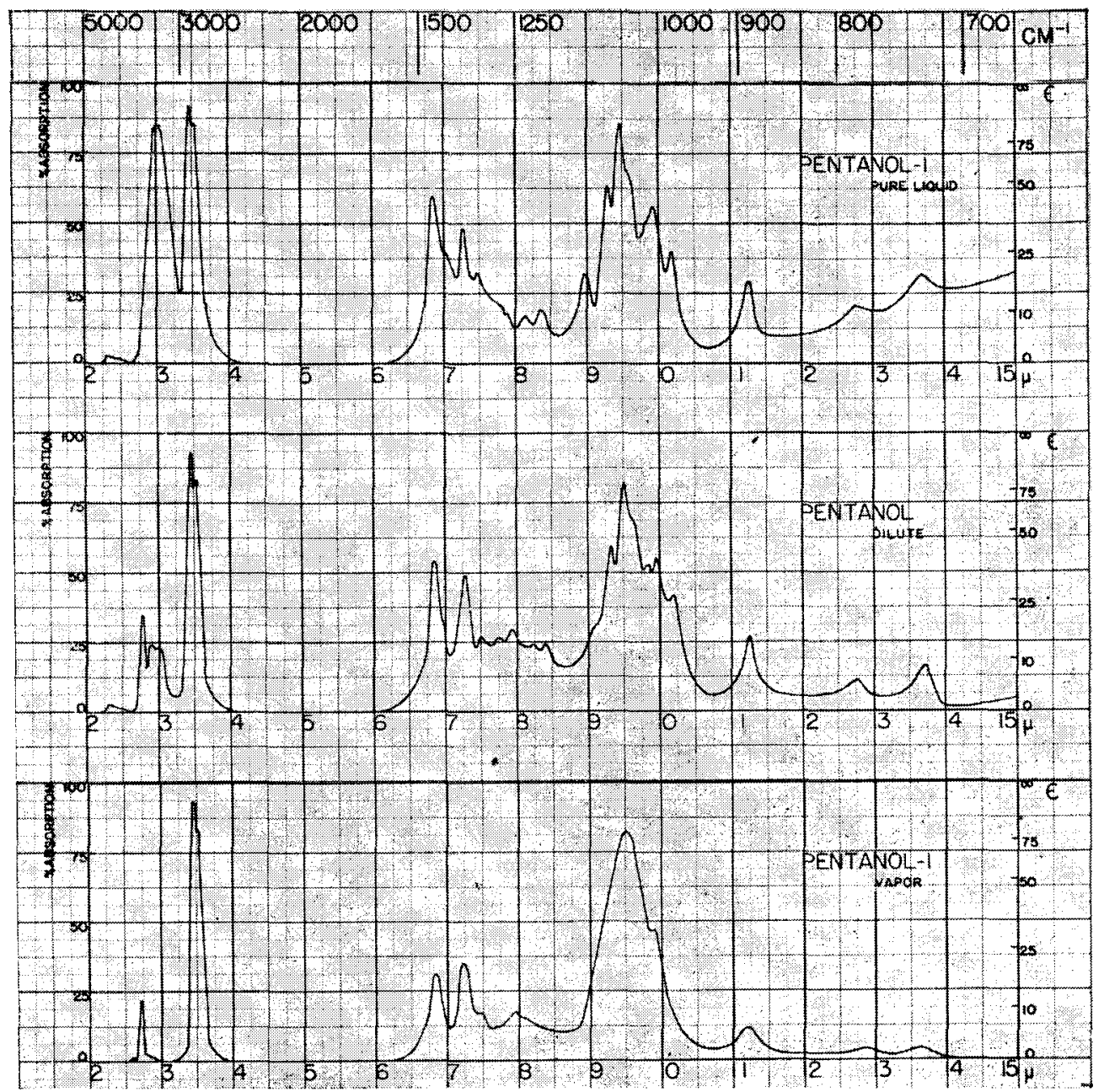

monomer band on its low-frequency edge shows a similar behavior, appearing at $890 \mathrm{~cm}^{-1}$ in $D$-ethanol and at $930 \mathrm{~cm}^{-1}$ in $D$-hexanol-1. The monomer band at $1240 \mathrm{~cm}^{-1}$ in ethanol and hexanol-1 disappears on deuteration in the same way as the monomer band at $1340 \mathrm{~cm}^{-1}$ in methanol. There is a weak narrow association band in $D$-ethanol and $D$-hexanol near 1135 $\mathrm{cm}^{-1}$. Between 1000 and $1100 \mathrm{~cm}^{-1}$ it is very difficult to be certain what is happening because of the intense absorption band common to all n-primary alcohols centered near $1050 \mathrm{~cm}^{-1}$, but it appears that the association band near $1100 \mathrm{~cm}^{-1}$ in ethanol is considerably weakened by deuteration and disappears from the spectrum of hexanol-1 on deuteration. The monomer bands near $1080 \mathrm{~cm}^{-1}$ and $1020 \mathrm{~cm}^{-1}$ in ethanol and hexanol (and at $1080 \mathrm{~cm}^{-1}$ in methanol) also appear to be removed by deuteration but they may be present with their intensity considerably reduced. The $470 \mathrm{~cm}^{-1}$ band was not investigated for $D$-ethanol and $D$ hexanol-1.

It should be added that additional effects due to association were noted in a few of the alcohols. In certain cases dilution caused a band maximum to shift.
Thus in ethanol a sharp band maximum at $1380 \mathrm{~cm}^{-1}$ shifts to $1390 \mathrm{~cm}^{-1}$ on dilution. It is not certain whether this means these are distinct association and monomer bands or this shift is due to the underlying wide association band. Since no consistent pattern was observed for minor effects of this kind, we shall not discuss them further in this paper.

\section{DISCUSSION}

\section{General Features}

Since it is well established that the stretching vibration of the $\mathrm{OH}$ group is separable from the stretching vibrations of the $\mathrm{CH}$ groups in all alcohols, let us start with the assumption that the two deformation vibrations of the $\mathrm{OH}$ group are also separable normal modes. If this is the case, then we may expect to find the following features in the spectra of all alcohols in the region between 1500 and $400 \mathrm{~cm}^{-1}$.

(1) The in-plane deformation vibration $\delta_{\text {Он }}$ should give rise to a band common to all alcohols somewhere between 1500 and $1000 \mathrm{~cm}^{-1}$. This band should be broad and diffuse in the pure alcohols and in concentrated 


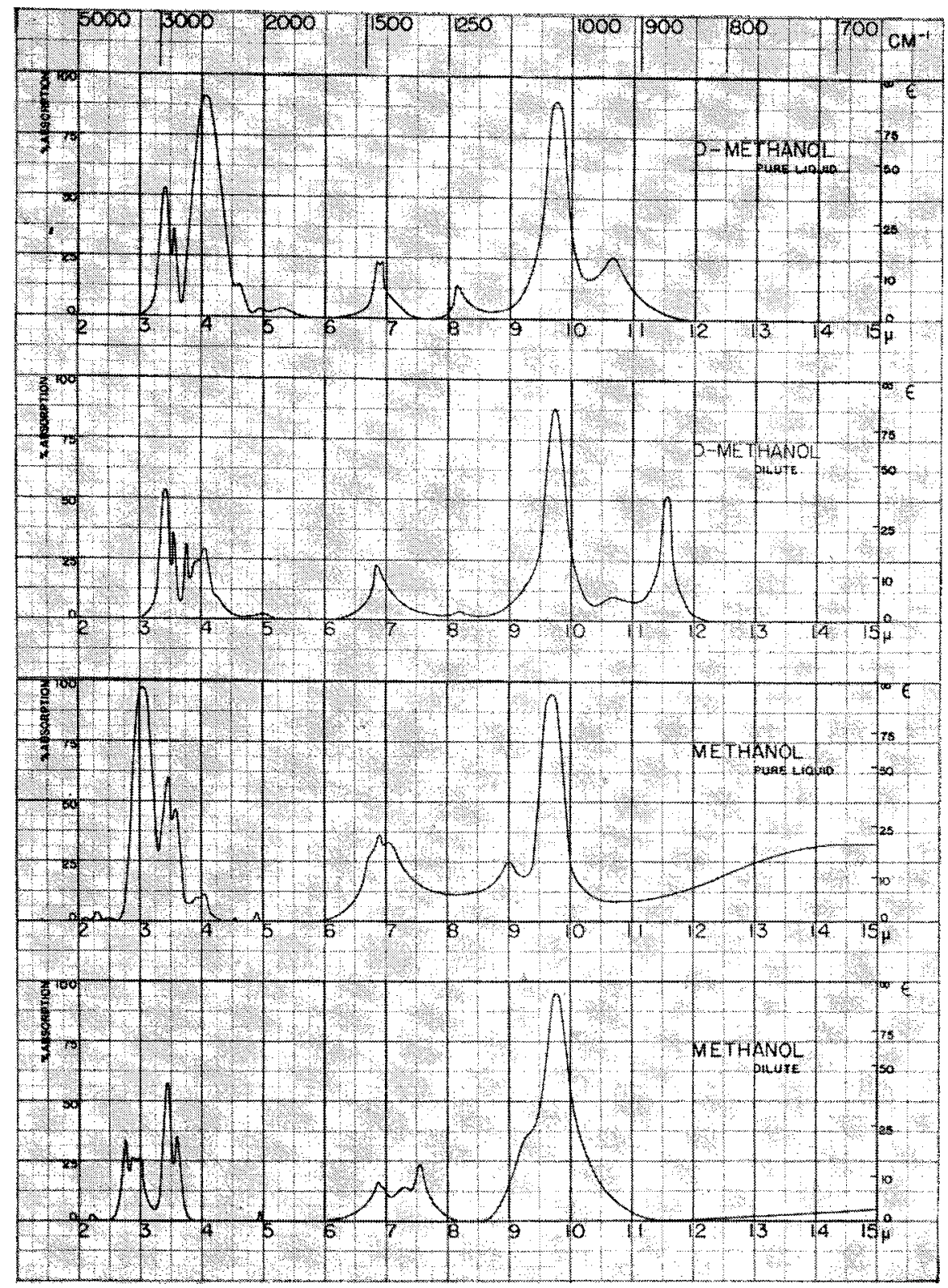

FrG. 7. Deuterated and undeuterated methanol as pure liquid, and as 0.1 molar solution in non polar solvent. solution but as dilution proceeds, it should weaken and be replaced by a narrow sharp band at a somewhat lower frequency. On deuterating the $\mathrm{OH}$ group, this pair of bands should disappear, being replaced by a similar pair whose frequencies are lower by a factor slightly less than $2^{\frac{1}{2}}$.

(2) The out-of-plane deformation vibration $\delta_{O H^{\prime}}$ should give rise to a similar pair of bands somewhere between 1000 and $400 \mathrm{~cm}^{-1}$.

In order to pick out such pairs of frequencies, the main results of the experiments on dilution have been summarized diagrammatically in Fig. 10 . If we first confine our attention to the region below $1000 \mathrm{~cm}^{-1}$, it is seen that a single wide association band common to all alcohols is found centered near $650 \mathrm{~cm}^{-1}$. This band disappears on dilution and on deuteration moves to 475 $\mathrm{cm}^{-1}$. It has already been assigned by us ${ }^{5}$ as the out-ofplane frequency, $\delta_{\mathrm{OH}}{ }^{\prime}$ and will not be discussed further in this paper.

In the region between 1500 and $1000 \mathrm{~cm}^{-1}$, where we might expect to find one association band with its corresponding monomeric band displaced to lower frequencies, we find in the primary and secondary alcohols 3 association bands close to 1410,1330 , and $1110 \mathrm{~cm}^{-1}$. In the 3 tertiary alcohols investigated, the $1410 \mathrm{~cm}^{-1}$ band is also present, but there is no sign of the $1330 \mathrm{~cm}^{-1}$ association band, and the $1110 \mathrm{~cm}^{-1}$ band appears to have moved up to between $1155 \mathrm{~cm}^{-1}$ and $1170 \mathrm{~cm}^{-1}$. It

A. V. Stuart and G. B. B. M. Sutherland, J. Chem. Phys. 20, 1977 (1952). 
should be added that in the primary alcohols, methanol is anomalous in that the $1330 \mathrm{~cm}^{-1}$ band is absent and instead a third association band is found at $1450 \mathrm{~cm}^{-1}$.

Although all the alcohols exhibit at least one monomeric band, there is at first sight no obvious correlation of the monomeric bands with the association bands. Thus one might have expected a monomeric counterpart to the $1410 \mathrm{~cm}^{-1}$ band which would be equally invariable in frequency over the whole range of alcohols. Such is definitely not the case. There is a monomeric band on the low-frequency side of the $1110 \mathrm{~cm}^{-1}$ band, i.e. near $1080 \mathrm{~cm}^{-1}$, in all of the secondary and branched primary alcohols. In the 3 tertiary alcohols there is a similar monomer band at $1145 \mathrm{~cm}^{-1}$ on the low-frequency side of an association band which varies in position from 1155 to $1195 \mathrm{~cm}^{-1}$. A similar monomeric band in the $n$-primary alcohols could have escaped detection if it lay between 1000 and $1070 \mathrm{~cm}^{-1}$, since all $n$-primary alcohols have an intense absorption in this region, conventionally assigned to the $\mathrm{C}-\mathrm{O}$ stretching frequency. There is actually definite evidence of such a band near $1080 \mathrm{~cm}^{-1}$ in some of our curves for methanol, ethanol, and hexanol. In the latter two cases, it is possible that there is also a weak monomeric band near $1020 \mathrm{~cm}^{-1}$.

The only other monomeric band common to all the alcohols is one which occurs somewhere between 1200 and $1320 \mathrm{~cm}^{-1}$. As seen from Fig. 10, it is extremely variable in position even within closely related series of alcohols. In methanol and in the 3 tertiary alcohols, it has an exceptionally high frequency $\left(1310\right.$ to $\left.1325 \mathrm{~cm}^{-1}\right)$.

The effects of deuteration may now be considered. These are illustrated in Fig. 11 for methanol, ethanol,

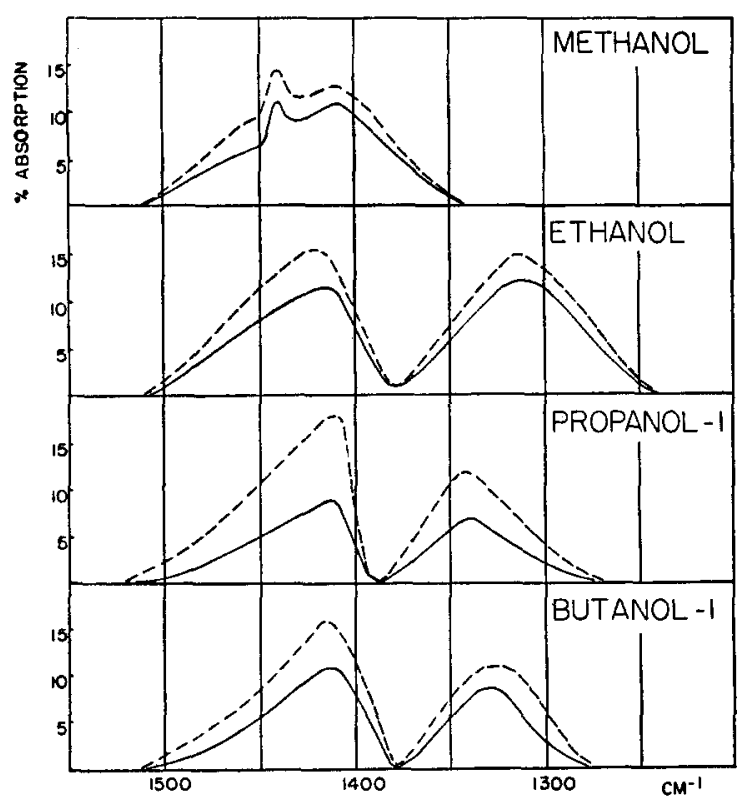

Frg. 8. Double compensation; cells 0.1 and $0.8 \mathrm{~mm}$; concentrations: -0.80 molar and 0.10 molar; $--\ldots 1.2$ molar and 0.15 molar.
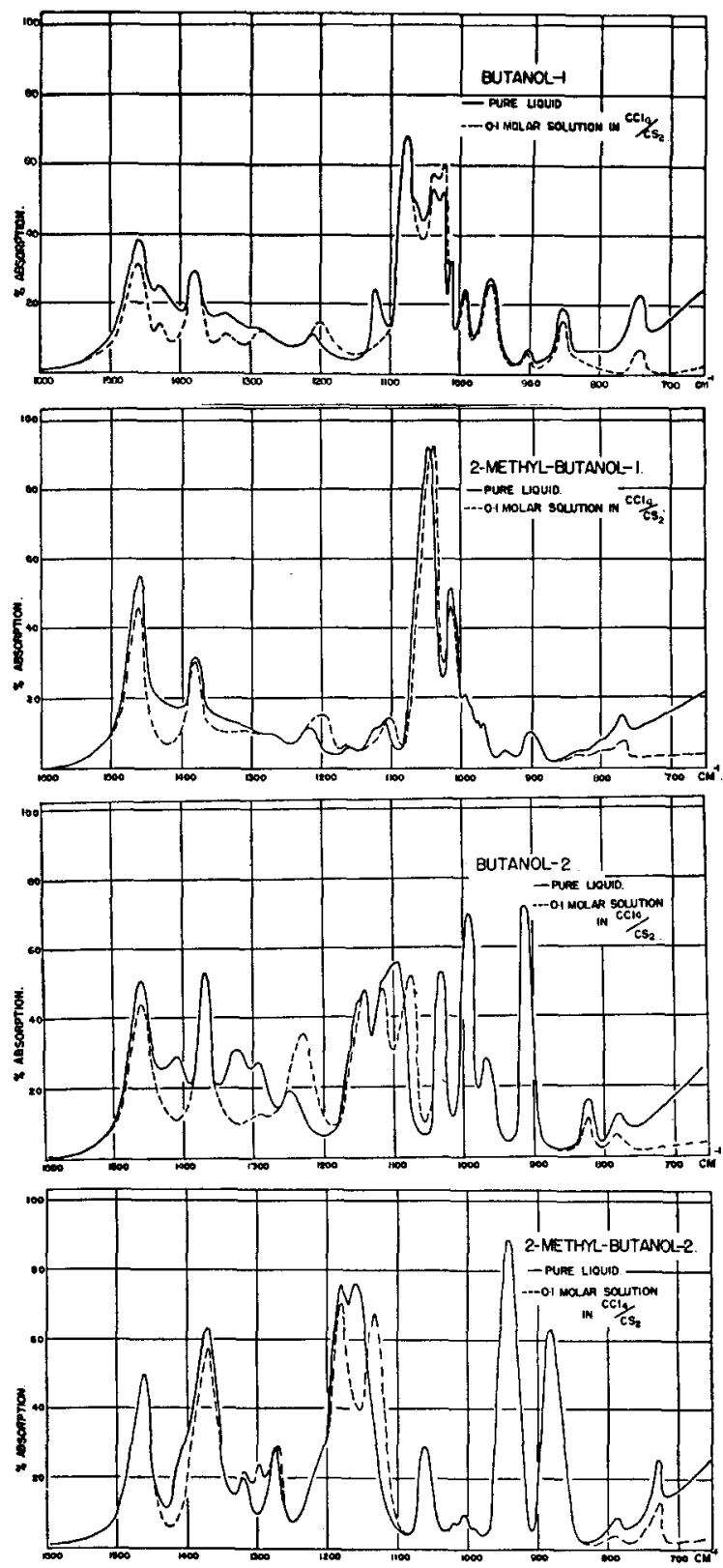

FIG. 9. Four butanols as $(-)$ pure liquid, and $(---) 0.1$ molar solution in non polar solvent; (a) butanol-1, (b) 2-methylbutanol-1, (c) butanol-2, (d) 2-methyl-butanol-2.

hexanol-1,"and phenol, the data for phenol being taken from the literature. ${ }^{6,7}$ In this diagram we have included the effects of dilution on the corresponding undeuterated alcohol. It will be observed that deuteration of the $\mathrm{OH}$ group consistently gives rise to a well-defined monomeric band near $900 \mathrm{~cm}^{-1}$. In the first 3 alcohols there is a corresponding association band near $950 \mathrm{~cm}^{-1}$ which shows the characteristic wide diffuse structure of $\mathrm{OH}$ association bands. No data are available on deuterated

${ }^{6}$ Williams, Hofstadter, and Herman, J. Chem. Phys. 7, 802 (1939).

7 M. M. Davies, J. Chem. Phys. 16, 267 (1948)! 


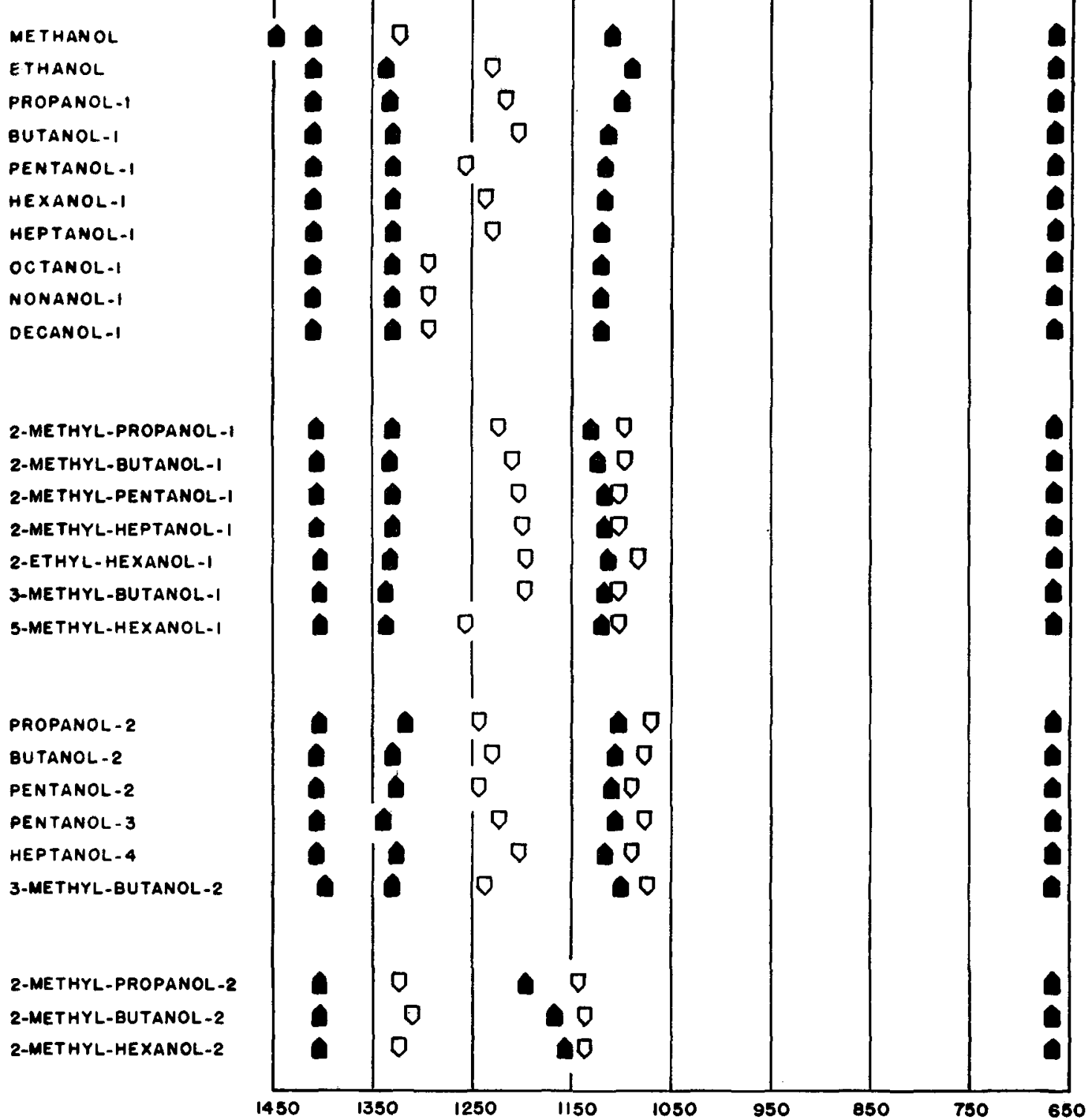

Frg. 10. Diagrammatic representation of the association (solid symbol), and monomer (open symbol) bands in 24 alcohols between 1500 and $650 \mathrm{~cm}^{-1}$.

phenol in the associated state, so it must not be assumed that it has no association band in this region.

\section{Assignment of the $\delta_{\mathrm{OH}}$ (in-plane) Frequency}

From the foregoing discussion, it may be concluded that in the region between 1500 and $1000 \mathrm{~cm}^{-1}$, the majority of undeuterated aliphatic alcohols possess two association bands and two corresponding dilution bands (as indicated in Table I). The higher frequency association band has a doublet structure, each component of which is wide and diffuse.

In the deuterated alcohols only one association band appears to be common to the alcohols investigated, viz. the $950 \mathrm{~cm}^{-1}$ band with a corresponding monomeric band near $900 \mathrm{~cm}^{-1}$. The problem is now to decide from these data the assignment of the $\mathrm{OH}$ in-plane deformation frequency. It will be found from the literature that there has been a good deal of controversy on this assignment. Herzberg, ${ }^{8}$ Noether, ${ }^{9}$ and Sutherland ${ }^{2}$ have favored the assignment of the $1370 / 1250 \mathrm{~cm}^{-1}$ complex to $\delta_{\mathrm{OH}}$, whereas Borden and Barker, ${ }^{10}$ Davies, ${ }^{7}$ and Quinan and Wiberly ${ }^{11}$ have preferred to assign this vibration to the $1110 / 1080 \mathrm{~cm}^{-1}$ complex. Good arguments have been put forward for each of these assign-

${ }^{8}$ G. Herzberg, The Infrared and Raman Spectra of Polyatomic Molecules (D. Van Nostrand Company, Inc., New York, 1945), p. 334 .

9 H. D. Noether, J. Chem. Phys. 10, 693 (1942).

${ }^{10}$ A. Borden and E. F. Barker, J. Chem. Phys. 6, 553 (1938).

11 J. R. Quinan and S. E. Wiberley, J. Chem. Phys. 21, 1896 (1953); Anal. Chem. 26, 1762 (1954). 
ments and equally good arguments against each of them. The present results seem to weight the evidence very conclusively in favor of the first assignment.

There are three strong arguments for assigning the $1370 / 1250 \mathrm{~cm}^{-1}$ complex to the $\mathrm{OH}$ in-plane deformation frequency in aliphatic alcohols.

(1) The association band in this region is over 200 $\mathrm{cm}^{-1}$ wide and shows a doublet structure. The two other association bands near 3300 and $650 \mathrm{~cm}^{-1}$ are also spread over $200 \mathrm{~cm}^{-1}$ and frequently show a doublet structure. The association band near $1110 \mathrm{~cm}^{-1}$ is not appreciably wider than any of the bands due to $\mathrm{CH}$ and $\mathrm{C}-\mathrm{C}$ vibrations.

(2) On deuteration, one would predict that an $\mathrm{OH}$ deformation frequency would be lowered by a factor of between 1.3 and 1.4. Thus in the deuterated alcohols one would expect to find the $1410 \mathrm{~cm}^{-1}$ frequency lowered to between 1085 and $1000 \mathrm{~cm}^{-1}$, while the $1330 \mathrm{~cm}^{-1}$ component would be predicted to be somewhere between 1030 and $957 \mathrm{~cm}^{-1}$. Similarly, the monomeric OD frequency would be predicted to be in the region between 970 and $890 \mathrm{~cm}^{-1}$. The $960 / 900 \mathrm{~cm}^{-1}$ complex found in the 3 deuterated aliphatic alcohols investigated falls within these limits. The ratio of the frequency of the $\mathrm{OH}$ to the corresponding $\mathrm{OD}$ bands is given in Fig. 11 on the line joining the two bands in question. It will be noticed that this ratio lies between 1.29 and 1.39 for the monomeric bands on ethanol, hexanol, and phenol, but that for methanol an anomalous figure of 1.52 is obtained. This has been one of the arguments used against the present assignment. It appeared much stronger when no experimental data were available for ethanol and hexanol. It seems to us that methanol is an anomalous case and may well be so since in this first member of the series some coupling may occur between the $\delta_{\mathrm{OH}}$ frequency and some other frequency.

(3) The third argument concerns the change in frequency which occurs in going from the association to the monomeric band. For the stretching frequency near $3 \mu$, this change is of the order of $7 \%$; for the out-ofplane $\mathrm{OH}$ deformation frequency, it must be greater

TABLE I. Association and monomeric bands of alcohols between 1500 and $1000 \mathrm{~cm}^{-1}$

\begin{tabular}{|c|c|c|c|c|}
\hline & $\begin{array}{l}\text { Association } \\
\text { bands in } \\
\mathrm{cm}^{-1}\end{array}$ & Remarks & $\begin{array}{l}\text { Monomeric } \\
\text { bands in } \\
\mathrm{cm}^{-1}\end{array}$ & Remarks \\
\hline \multirow[t]{2}{*}{$\mathrm{R}-\mathrm{OH}$} & $1370 \begin{array}{l}(1410) \\
(1330)\end{array}$ & $\begin{array}{l}\text { Wide diffuse bands. } \\
1330 \text { component ab- } \\
\text { sent in methanol and } \\
\text { tertiary alcohols }\end{array}$ & 1250 & $\begin{array}{l}\text { Variable in posi- } \\
\text { tion. Found at } \\
1330 \text { in methanol } \\
\text { and tertiary al- } \\
\text { cohols }\end{array}$ \\
\hline & 1110 & $\begin{array}{l}\text { Narrow band. Found } \\
\text { near } 1155 \text { in tertiary } \\
\text { alcohols }\end{array}$ & 1080 & $\begin{array}{l}\text { Not yet found in } \\
\text { all } n \text {-primary al. } \\
\text { cohols. Appears at } \\
1145 \text { in tertiary } \\
\text { alcohols }\end{array}$ \\
\hline $\mathrm{R}-\mathrm{OD}$ & 950 & Wide diffuse band & 900 & $\begin{array}{l}\text { Variable in posi- } \\
\text { tion from } 870 \text { to } \\
930 \mathrm{~cm}^{-1}\end{array}$ \\
\hline
\end{tabular}

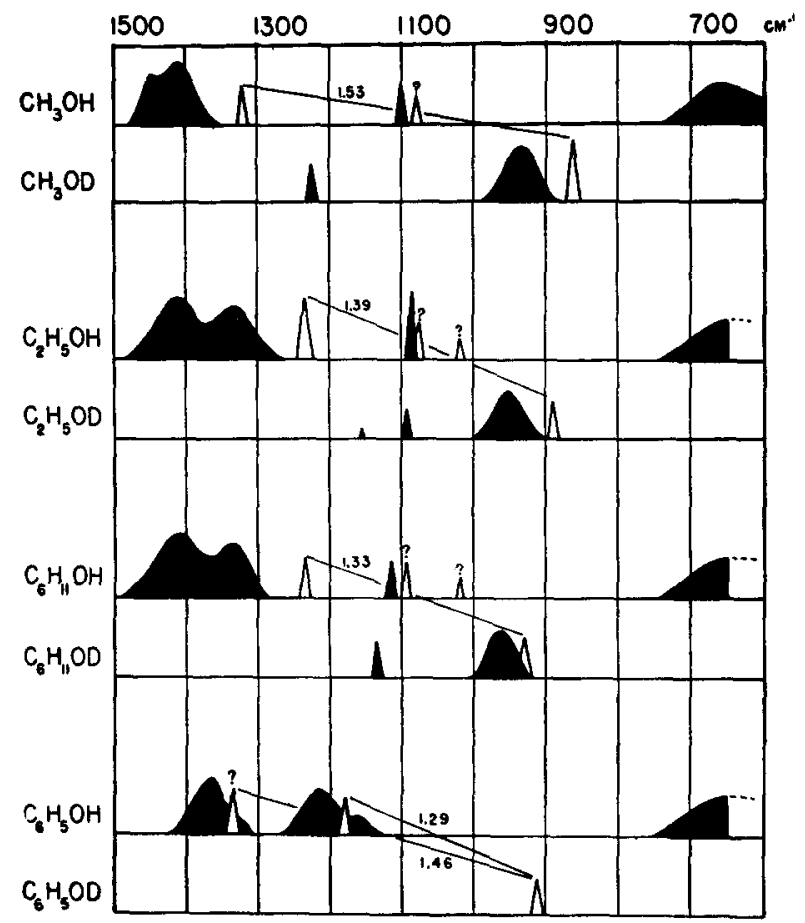

FIG. 11. The association (shaded) and monomeric (unshaded) bands of some deuterated and undeuterated alcohols.

than $10 \%$ since we have not been able to identify the monomeric frequency in solution. The change for the in-plane $\mathrm{OH}$ deformation frequency would therefore be expected to be not less than $10 \%$ since the effect of the hydrogen bonding forces is likely to be greater on deformation frequencies than on stretching frequencies where the unperturbed force constants are rather high. The mean shift in the $1370 / 1250 \mathrm{~cm}^{-1}$ complex is exactly $10 \%$, whereas the mean shift in the $1110 / 1080$ $\mathrm{cm}^{-1}$ complex is only $3 \%$.

There does not appear to be any simple interpretation of the doublet structure in the $1370 \mathrm{~cm}^{-1}$ association band. This doubling must have a cause different from the doubling observed in the $\mathrm{OH}$ stretching band at $3450 \mathrm{~cm}^{-1}$ for the following reasons. In the latter case, the two components show a very different dependence on concentration, whereas the 1330 and $1410 \mathrm{~cm}^{-1}$ bands show essentially the same dependence on concentration (Fig. 8). Furthermore, all alcohols in solution in nonpolar solvents show a doublet near $3450 \mathrm{~cm}^{-1}$, whereas some alcohols do not show the $1410 / 1330 \mathrm{~cm}^{-1}$ doublet (Fig. 10). It will be noted that the exceptions are the tertiary alcohols and methanol (if we ignore the anomalous band in the latter at $1445 \mathrm{~cm}^{-1}$ ). This suggests that when there is a symmetric or approximately symmetric potential field about the $\mathrm{CO}$ bond, to which the $\mathrm{H}$ is attached, no doubling is observed. It seems, therefore, that steric effects and rotational isomerism may be quite important in unravelling some of the complexities of the spectra of associated alcohols. 


\section{The Association and Monomer Bands near $1100 \mathrm{Cm}^{-1}$}

Accepting the assignment of $\delta_{\mathrm{OH}}$ in the preceding section, the explanation of the association and monomer bands near $1100 \mathrm{~cm}^{-1}$ must now be considered. It seems probable that these bands are to be associated in some way with the intense band found in all $n$-primary alcohols near $1050 \mathrm{~cm}^{-1}$. In methanol the latter band has been unequivocally assigned to the $\mathrm{C}-\mathrm{O}$ stretching vibration. Although in other alcohols a similar assignment is conventionally made, it is unlikely that the $\mathrm{C}-\mathrm{O}$ stretching frequency is separable since the coupling of such a frequency would be very strong to similar $\mathrm{C}-\mathrm{C}$ frequencies. This is borne out by the observation that in other primary alcohols there is usually not a single band near $1050 \mathrm{~cm}^{-1}$ but several intense bands between $1000 \mathrm{~cm}^{-1}$ and $1100 \mathrm{~cm}^{-1}$. We shall therefore refer to these absorption bands as $-\mathrm{C}-\mathrm{O}-\mathrm{C}-\mathrm{C}-$ vibrations. It has been noted that this group of bands moves towards somewhat higher frequencies in secondary alcohols and still higher frequencies in tertiary alcohols $\left(1175 \mathrm{~cm}^{-1}\right)$. It is significant that the corresponding association and monomeric bands in tertiary alcohols are also found at appreciably higher frequencies (Fig. 10).

Although the possibility that these bands are due to $\mathrm{CH}$ deformation motions cannot be excluded, the most reasonable conclusion is that the association and monomer bands between 1000 and $1200 \mathrm{~cm}^{-1}$ are somehow connected with the effects of association on the $-\mathrm{C}-\mathrm{O}-\mathrm{C}-\mathrm{C}-$ vibrations. The exact nature of this interaction effect is not yet understood. It is important to note that the most intense bands in the neighborhood of $1050 \mathrm{~cm}^{-1}$ in the primary alcohols appear to be affected very little by association. In the secondary alcohol, butanol-2 (where the $-\mathrm{C}-\mathrm{O}-\mathrm{C}-\mathrm{C}-$ complex of bands is probably near $1100 \mathrm{~cm}^{-1}$ ) there is a marked shift of the most intense band to lower frequencies on dilution and the same phenomenon is observed in the tertiary alcohol, 2-methyl-butanol-2, for the band at $1150 \mathrm{~cm}^{-1}$. This is consistent with the above explanation since the coupling of the $\mathrm{C}-\mathrm{O}$ and $\mathrm{C}-\mathrm{C}$ vibrations will be much greater in secondary and tertiary alcohols than in the primary ones where the $\mathrm{OH}$ group is merely tacked on to the end of a $\mathrm{C}-\mathrm{C}-\mathrm{C}$ chain.

\section{General Conclusions}

Several general conclusions may be drawn from this work. The first is that all aliphatic alcohols show a wide diffuse association band centered near $650 \mathrm{~cm}^{-1}$, which can be assigned to the out-of-plane deformation vibration of the $\mathrm{H}$ atom in the $\mathrm{COH}$ group. The corresponding monomeric band has not been observed, except in gaseous methyl alcohol, where it is really a hindered rotation, ${ }^{12}$ although it can be loosely described as a libration with a mean frequency of about $225 \mathrm{~cm}^{-1}$. This vibration appears to be a separable fundamental, i.e. it resembles the $\mathrm{OH}$ stretching frequency in that the motion of the $\mathrm{H}$ atom is virtually independent of the rest of the molecule.

The second general conclusion is that the other deformation motion of the $\mathrm{H}$ atom (i.e. in the plane of the $\mathrm{COH}$ group) is also a fairly well-defined separable mode of vibration. In associated aliphatic alcohols it consistently gives rise to a very broad band extending from 1300 to $1500 \mathrm{~cm}^{-1}$ which on deuteration gives a broad band centered near $930 \mathrm{~cm}^{-1}$. The monomeric value of this frequency is quite variable in undeuterated alcohols (1200 to $1330 \mathrm{~cm}^{-1}$ ) but appears to be less variable $\left(870\right.$ to $\left.930 \mathrm{~cm}^{-1}\right)$ in deuterated alcohols. This variation may be due to some coupling of the $\mathrm{OH}$ inplane deformation motion with a skeletal or hydrogenic motion and this effect seems to be quite marked in methyl alcohol. It may be, however, that the force constant controlling this vibration varies from one alcohol to another since the corresponding OD deformation frequency also varies in magnitude from one alcohol to another.

The other conclusions are that (a) the skeletal frequencies of the alcohol molecule involving stretching of the $\mathrm{C}-\mathrm{O}$ band are affected by hydrogen bonding; (b) steric effects and rotational isomerism may be important factors in elucidating the spectra of associated alcohols; and (c) some of the $\mathrm{CH}$ deformation frequencies may be affected by hydrogen bonding through coupling with $\mathrm{OH}$ deformation frequencies or skeletal modes involving stretching of the $\mathrm{C}-\mathrm{O}$ bond.

\section{ACKNOWLEDGMENTS}

The authors wish to acknowledge the very valuable help received from Dr. Gilbert J. Sloan who purified many of the alcohols for them and also prepared the deuterated alcohols. They also wish to thank Sharples Chemicals, Inc. for the gift of pure samples of 7 pentanols. To Professor W. R. Vaughan they are most indebted for much valuable advice on purification and for the actual purification of 2-methyl-pentanol-1. Mr. Martin Harwit did some valuable confirmatory runs on the spectra of ethanol and $D$-ethanol. One of us (A.V.S.) wishes to express his appreciation of a Fellowship from the Dow Chemical Company of Midland, Michigan, during the tenure of which a very significant portion of this work was accomplished.

${ }^{12}$ D. C. Burkhard and D. M. Dennison, Phys. Rev. 86, 108 (1951). 